\title{
Transforaminal Lumbar Interbody Fusion (TLIF) Technique for the Treatment of Degenerative Lumbar Disease
}

G.E.Kazim, A.I.Ali, M.M.El Karamany and M.S.Mohammed

Orthopedic Surgery Dept., Faculty of Medicine, Benha Univ., Benha, Egypt

E-Mail: Mostafa11@gmail.com

\begin{abstract}
Transforaminal lumbar interbody fusion (TLIF) is a relatively new technique of lumbar arthrodesis via posterior transforaminal approach to the disc, indicated mainly in cases of degenerative disc disease, low grade spondylolisthesis and reoperation for disc herniation, especially when there is indication for interbody fusion and posterior decompression. The aim of the study is to asses and evaluates the outcome of posterior instrumeneted transforaminal lumbar interbody fusion as a surgical treatment modality for symptomatic lumbar degenerative disease. this was a clinical trial study, including 20 cases of symptomatic degenerative lumbar disease who have failed medical treatment which includes one or more of the following: more than 40-year-old, having radiological evidence of lumbar degenerative disease whether stable or unstable. The duration of the study ranged from 6-12 months. Oswestry Disability Index (ODI) at baseline was ranged between $42-80$ with a mean value of $61.65 \pm 11.380$ and it was decreased significantly after 1 year of surgery with a mean value of $35.35 \pm 11.417$. VAS score at baseline was ranged between $4-9$ with a mean value of $6.80 \pm 1.609$ and it was decreased significantly after 1 year of surgery with a mean value of $0.75 \pm 0.910$. TLIF is a technique which offers a simple, safe and effective treatment for degenerative lumbar spine disorders with great improvement of life quality of cases with surgery satisfaction.
\end{abstract}

Keywords: Transforaminal Lumbar Interbody Fusion, Lumbar Disc Disease, Oswestry Disability Index, Degenerative Lumbar Spine.

\section{Introduction}

Interbody fusion techniques have been developed to preserve the load-bearing capacity of the spine, restore sagittal plane alignment and proper disc height, all of which enhance the potential for fusion. The unilateral transforaminal approach for segmental lumbar interbody fusion was first described in the 1980s by [1] and it was popularized in the late 1990s by Harms and Jeszensky [2].

Transforaminal lumbar interbody fusion (TLIF) is an alternative technique, which avoids the anterior approach and the approach through the spinal canal. Theoretically, it prevents typical complications, such as those seen in anterior and posterior lumbar interbody fusion [3].This approach offers the advantage that it can easily be performed unilaterally. This results in less destruction of the posterior elements and less gross destabilization of the spine, which will maximize fusion stability. Furthermore, it allows better access to the neuroforamen and reduces the need to manipulate spinal nerve roots. Thus, nerve injuries that may occur during retraction may be avoided.

For those with lumbar stenosis but without spondylolisthesis (deformity), the surgical management has traditionally involved posterior decompressive procedures, including laminectomy or laminotomy, and judicious use of partial medial facetectomies and foraminotomies, with or without discectomy. In patients with evidence of spinal instability, however, in situ posterior lumbar fusion is recommended as a treatment option in addition to decompression in the setting of lumbar stenosis [4].

Secondary indications include recurrent lumbar disc herniation, where extensive bony removal is necessary for exposure of the disc fragments, lateral or massive disc herniations, failed previous lumbar fusions by other techniques, and discogenic low back pain [5].

Although most cases of low back pain are transient and relieved by comfort measures along with temporary activity modification, conservative management remains ineffective in approximately $5 \%$ of cases that go on to become chronic and disabling [6], resulting in a need for more aggressive treatment.

Lumbar spinal fusion may be used as a potential adjunct, however, in patients with a herniated disc in whom there is evidence of preoperative spinal deformity. Because lumbar deformity, instability, or even chronic low back pain may occur as a result of a reoperative lumbar discectomy, fusion is often considered in the setting of repeated lumbar disc herniations [4].

\section{Subjects and methods}

The study had been conducted at Benha university hospital, orthopedics department and Royal national orthopaedic hospital stanmore london.

\subsection{Study design}

This is an intervention type of epidemiologic studies termed retrospective study with randomized controlled clinical trial that had been conducted a long twelve months.

\subsection{Target population}

Symptomatic degenerative lumbar disease patients who have failed medical treatment.

\subsection{Study population}

Symptomatic degenerative lumbar disease patients who have failed medical treatment attending benha university hospital.

\subsection{Inclusion criteria}

This study had been included 20 cases of symptomatic degenerative lumbar disease who have failed medical treatment which includes one or more of the following: more than 40 year old and Having radiological evidence of 
lumbar degenerative disease whether stable or unstable.

\subsection{Exclusion criteria}

Any cases with the following criteria will be excluded: Incomplete radiological documentation, inaccurate radiological documentation before or after the TLIF and Anticipated poor cooperation of the patient.

\subsection{Sampling technique}

Random allocation of forty above 40 years old, symptomatic degenerative lumbar disease patients, who have failed medical treatment attending benha university hospital and Royal national orthopaedic hospital, Twenty of them meet the inclusion criteria and are recruited for the study group, the rest will be the control group.

Sample size: Case group: Twenty symptomatic degenerative lumbar disease patients who have failed medical treatment and give consent to be subjected to surgical intervention aged above 40 years old.

\subsection{Control group}

comparable number of symptomatic degenerative lumbar disease patients who had failed medical treatment and don't give consent to be subjected to surgical intervention aged above 40 years old.

\subsection{Methods of diagnosis}

All patients were evaluated clinically by history and physical examination. Special attention is directed towards associated neurology, previous spine procedures, gait disturbance, and any change in body habitus and posture. All the patients had standing radiographs of the lumbar spine (Anteroposterior and lateral views). Taking care of the radiological signs of frank instability (lithesis more than $3.5 \mathrm{~mm}$ or cobbs angle more than 10 degrees).

\section{Assessment and outcome evaluation had been included}

Radiological evaluation, Clinical outcomes are assesed by the patients using the Oswestry Disability Index (ODI) and visual analog scale (VAS) questionnaires, Patient stastifaction and clinical improvement and Presence of complications.

\subsection{Surgical technique}

Antibiotics were given intravenously at induction of anesthesia and for 3 day postoperatively. Then oral antibiotics for 10 post operatively.

\subsection{0perative details}

-Date of operation - Operative time - Theatre -Blood loss - Anesthesia - Approach - Soft tissue and muscle condition

\section{STEPS:0 /58}

\section{Step 1-Pedicle screws insertion}

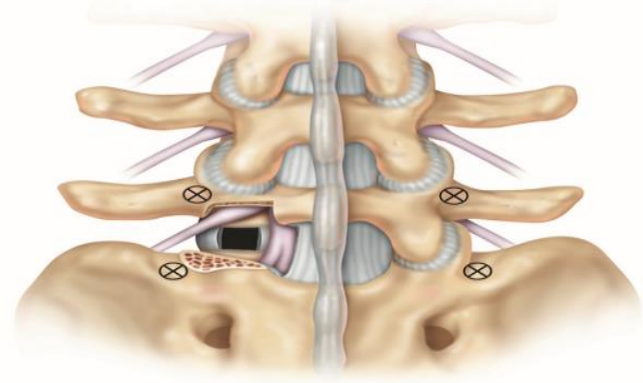

Fig (1) Identify pedicle insertion points. The optimal insertion point is at the intersection of the transverse process and pars interarticularis [7].

\section{Step 2 Facetectomy and working Zone Preparation (L5/S1)}

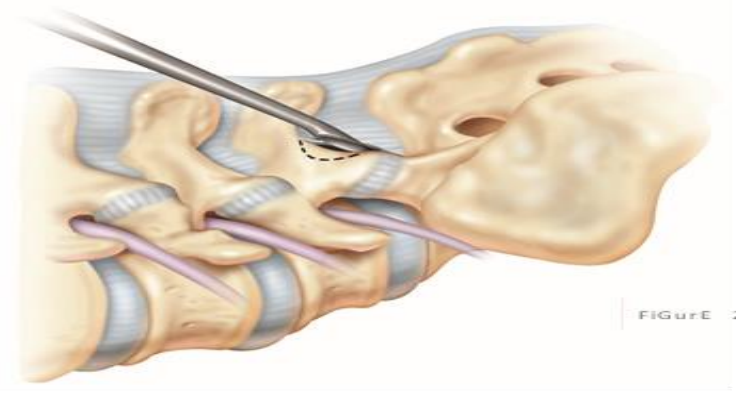

Fig (2) The inferior lamina of L5 can be removed by a kerrison rongeur illustrated by the dotted line [7].

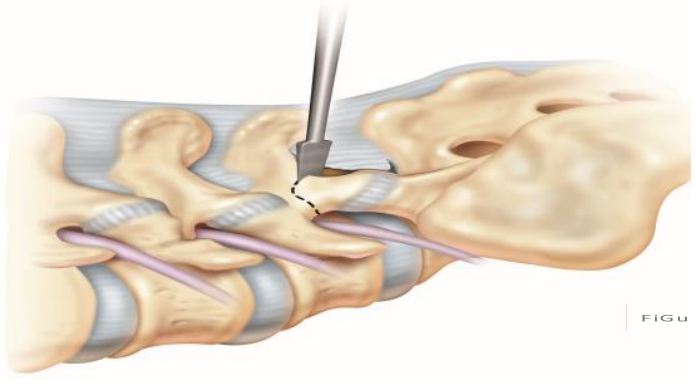

Fig (3) Resect the inferior articular process of L5 with a straight osteotome or a Kerrison

[7]. 


\section{Step 3: Annulotomy and Initial Disc Dissection}

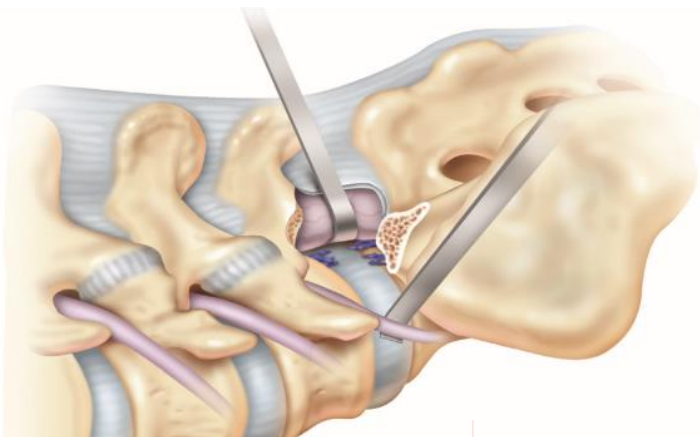

Fig (4) Dissector or nerve root retractoris used to ensure the protection of these structures at every step of the procedure [7].

\section{Step 4: Initial Distraction and Preparation of Disc Space}

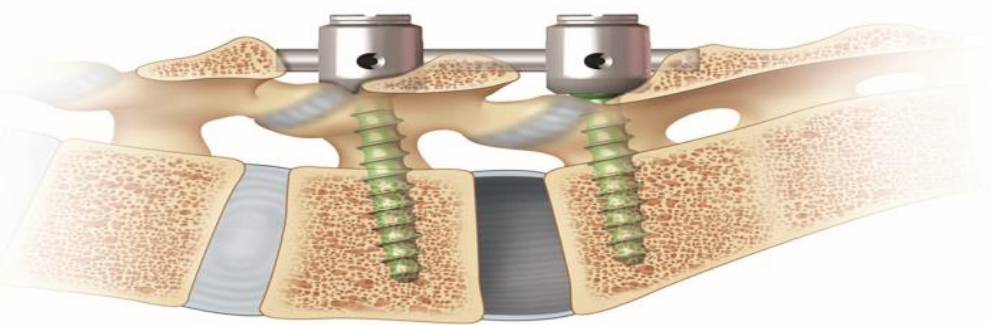

Fig (5) Once distraction is obtained, the opening of the disc space can be maintained with either a temporary rod or the use of a laminar spreader between the spinous processes [7].

\section{Step 5: Final Disc Preparation and Endplate Cleaning}

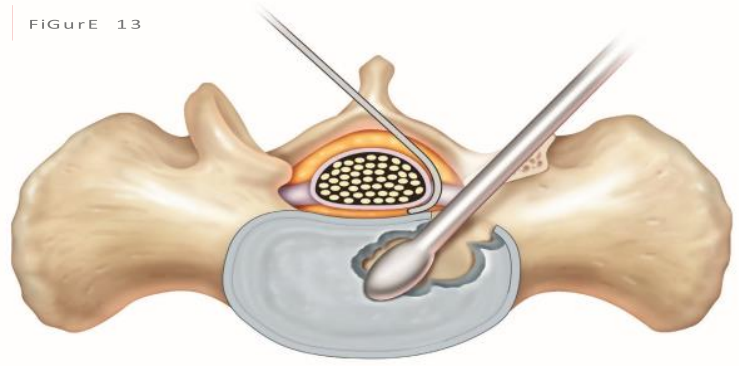

Fig (6) The final discetomy is performed using a combination of curettes, osteotomes, rongeurs and shavers [7].

\section{Step 6: Decortication and Placement of Bone Graft}

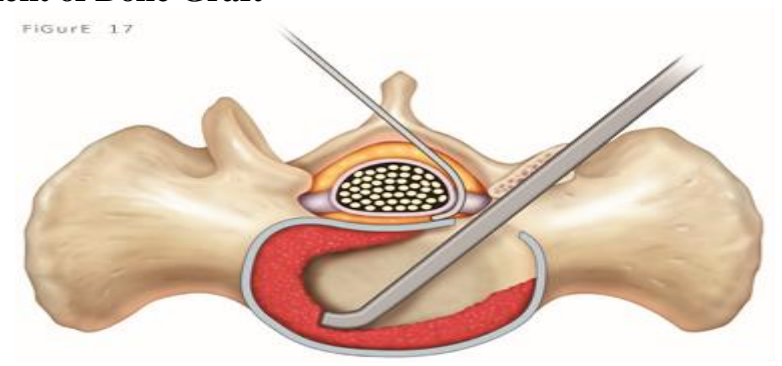

Fig [7] In order to achieve a solid interbody fusion, the disc space should be filled with as much bone graft as possible. Fill the anterior third and contra-lateral side of the disc space with bone graft using a variety of straight and curved bone tamps from the disc preparation set [7]. 


\section{Step 7: Cage Trialing}

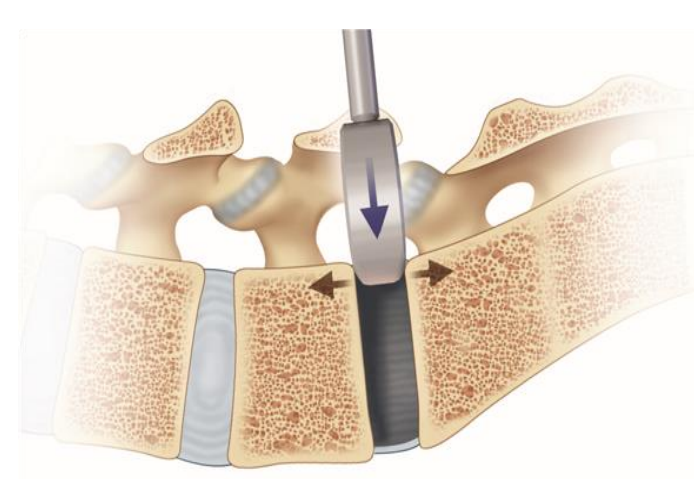

Fig (8) A cage trial should be used prior to insertion of the implant to evaluate potential cage placement and determine the optimal implant fit [7].

\section{STEP 8: Cage Insertion - Concorde Bullet}

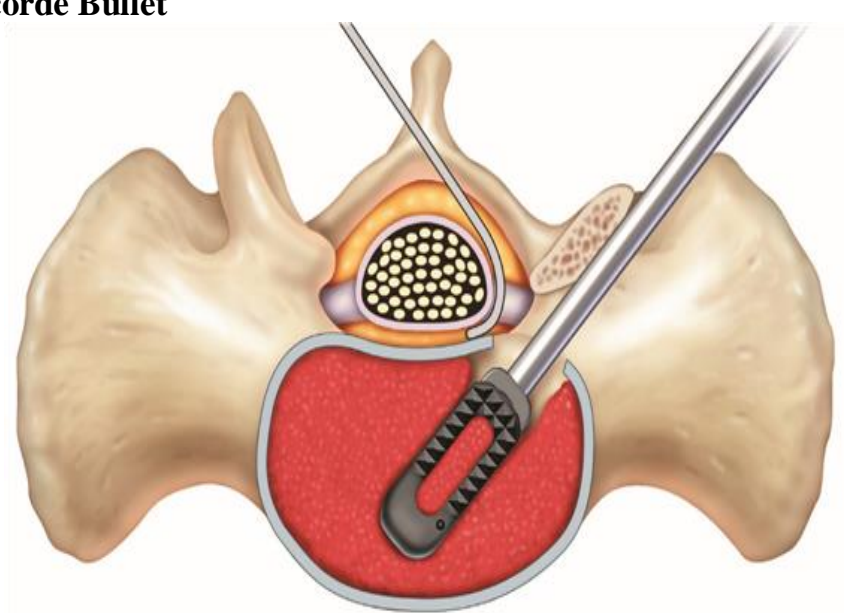

Fig (9) Align threaded hole of cage with threaded tip. Tighten the knob clockwise until cage is secure. Take care not to cross thread or overtighten the inserter [7].

STEP 9: Implant orientation for lordotic CONCORDE Bullet

Step 10: Final Compression

STEP 11: Verification of final cage placement - concorde Bullet

An X-ray should be taken to verify final cage placement.
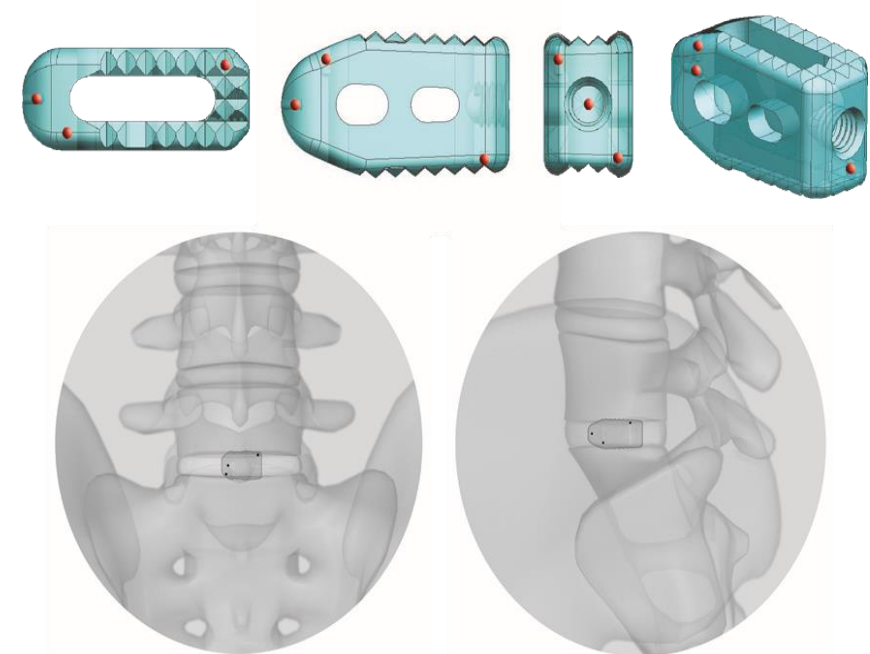

Fig (34) The appearance of three tantalum beads will identify the position of the CONCORDE Bullet cage in the sagittal, coronal and axial planes [7]. 


\section{Postoperative care}

The patients are admitted to the hospital the patients receive intravenous antibiotics, pain medication as required.

The patient is typically mobilized out of bed the day after surgery.

\section{Follow up}

Patients were asked to return to hospital for follow-up at 4 weeks, 3 months, 6 months, 12 months, and thereafter once a year after operation.

\section{Administrative design}

- Approvales: -An informed verbal consent from all participants was taken and confidentiality of information was assured. -An official written administrative permission letter was obtained from dean of faculty of medicine, Benha university hospital. The title and objectives of the study were explained to them to ensure their cooperation.

- Ethical committee: Permission from the faculty of medicine ethical committee was also obtained and approval from institutional review board was taken.

\section{Results}

Table (1) shows TLF level of the studied group show that degenerated segment was L4/L5 in 20(100\%), degenerated segment was L5/S1 in $5(25 \%)$ and $3(15 \%)$ had in multi levels.

Table (2) show operation data results. Surgical time was ranged between 1.5 - 3.5 hours with a mean value of $2.60 \pm 0.620$ hours. Blood loss was ranged between $160-$ $345 \mathrm{ml}$ with a mean value of $248.75 \pm 62.993 \mathrm{ml}$. Hospital stay was ranged between $4-7$ with a mean value of $5.95 \pm 1.146$.

Table (3) show assessment tools results. Oswestry Disability Index (ODI) at bassline was ranged between 42 - 80 with a mean value of $61.65 \pm 11.380$ and it was decreased significantly after 1 year of surgery with a mean value of $35.35 \pm 11.417$. VAS score at bassline was ranged between $4-9$ with a mean value of $6.80 \pm 1.609$ and it was decreased significantly after 1 year of surgery with a mean value of $0.75 \pm 0.910$.

Table (4) shows Patient satisfaction of the studied group show that $17(85 \%)$ had good rate of satisfaction and $3(15 \%)$ had poor satisfaction.

Table (5) shows complications of the studied group show that $3(15 \%)$ had Infection, $2(10 \%)$ had Persistent back pain, 1(5\%) had Pseudarthrosis and 1(55) had Neurological deficit.

Table (6) shows Pain improvement of the studied group show that $18(90 \%)$ had pain improving and $2(10 \%)$ had no improving.

Table (1) Distribution of studied sample according to patient's TLF level.

\begin{tabular}{lcc}
\hline TLF Level & Number & Percent \\
\hline L4/L5 & 20 & 100 \\
L5/S1 & 5 & 25 \\
Multi levels & 3 & 15 \\
\hline
\end{tabular}

Table (2) Distribution of studied sample according to patient's operation data.

\begin{tabular}{lcc}
\hline & Min. - Max. & Mean \pm S.D. \\
\hline Surgical Time & $1.5-3.5$ & $2.60 \pm 0.620$ \\
Blood Loss & $160-345$ & $248.75 \pm 62.993$ \\
Hospital Stay & $4-7$ & $5.95 \pm 1.146$ \\
\hline
\end{tabular}

Table (3) Comparison between before and after treatment according to patient's assessment tools.

\begin{tabular}{lccc}
\hline & Before & After 1 year & P Value \\
Oswestry Disability Index (ODI) & & & \\
\hline Min. - Max. & $42-80$ & $21-65$ & $<0.001^{*}$ \\
Mean + S.D. & $61.65 \pm 11.380$ & $35.35 \pm 11.417$ & \\
VAS score & & & \\
Min. - Max. & $4-9$ & $0-3$ & $<0.001^{*}$ \\
Mean \pm S.D. & $6.80 \pm 1.609$ & $0.75 \pm 0.910$ & \\
\hline
\end{tabular}

Table (4) Distribution of studied sample according to patient's satisfaction.

\begin{tabular}{lcc}
\hline Patient satisfaction & Number & Percent \\
\hline Good & 17 & 85 \\
Poor & 3 & 15 \\
Total & 20 & 100 \\
\hline
\end{tabular}


Table (5) Distribution of studied sample according to patient's complications.

\begin{tabular}{lcc}
\hline Complications & Number & Percent \\
\hline Infection & 3 & 15 \\
Persistent back pain & 2 & 10 \\
Pseudarthrosis & 1 & 5 \\
Neurological deficit & 1 & 5 \\
\hline
\end{tabular}

Table (6) Distribution of studied sample according to patient's Pain improvement.

\begin{tabular}{lcc}
\hline \multicolumn{1}{c}{ Pain improvement } & Number & Percent \\
\hline Improve & 18 & 90 \\
No improving & 2 & 10 \\
Total & 20 & 100 \\
\hline
\end{tabular}

\section{Discussion}

Approximately $70 \%$ to $85 \%$ of adults will be affected by low back pain (LBP) at some point during their lifetimes. Numerous anatomic sites can be responsible for the pain, and accurate diagnosis is often difficult. Degenerative disc disease (DDD), internal disc disruption, lumbar disc herniation, and facet joint arthritis, as well as intra-abdominal pathology, are all potential causes of LBP [7].

This is why this study was selected to be conducted to asses and evaluate the outcome of posterior instrumented transforaminal lumbar interbody fusion as a surgical treatment modality for symptomatic lumbar degenerative disease.

A clinical trial study was held, including 20 cases of symptomatic degenerative lumbar disease who have failed medical treatment which includes one or more of the following: more than 40-year-old, having radiological evidence of lumbar degenerative disease whether stable or unstable. The duration of the study ranged from 6-12 months.

The present study shows that TLF level of the studied group show that degenerated segment was L4/L5 in $20(100 \%)$ and degenerated segment was L5/S1 in 5(25\%).

[8] demonstrated that clinical presentations in their study were mechanical back pain in 100\% of cases and leg pain in $90 \%$ of cases. Female to male ratio was 1:4.

Lumbar spinal fusion was introduced approximately 70 years ago and has evolved as a treatment option for symptomatic spinal instability, spinal stenosis, spondylolisthesis, and degenerative scoliosis. Broader applications including use as a treatment of chronic low back pain and recurrent radiculopathy have resulted in a dramatic increase in the rates of lumbar fusion procedures within the last decade in the United States. Lumbar spinal fusion is often performed after a posterior decompressive procedure when there is evidence of preoperative lumbar spinal deformity or instability that could worsen after laminectomy alone [4].

In the study in our hands, surgical time was ranged between $1.5-3.5$ hours with a mean value of $2.60 \pm 0.620$ hours. Blood loss was ranged between $160-345 \mathrm{ml}$ with a mean value of $248.75 \pm 62.993 \mathrm{ml}$. Hospital stay was ranged between $4-7$ with a mean value of 5.95 \pm 1.146 . Oswestry Disability Index (ODI) at bassline was ranged between $42-80$ with a mean value of $61.65 \pm 11.380$ and it was decreased significantly after 1 year of surgery with a mean value of $35.35 \pm 11.417$. VAS score at bassline was ranged between $4-9$ with a mean value of $6.80 \pm 1.609$ and it was decreased significantly after 1 year of surgery with a mean value of $0.75 \pm 0.910$.

Our results are supported by study of [9] as they concluded that the TLIF showed to be a good alternative to PLIF with relatively less risk of complications, less operating time and hospitalization, as well as significant reduction in blood loss during operation. TLIF approach lessens the potential for nerve root injury, therefore resolving probably the most important limitation of the PLIF procedure. For this reason, and the case specific advantages of TLIF over a combined anterior and posterior single-level fusion, they favored TLIF over PLIF as the choice surgical procedure for the posterior operative management of symptomatic degenerative lumbar spine disorders.

Furthermore, [10] reported that Statistically significant differences in favor of minimally invasive treatment were immediate postoperative pain (VAS at third day after operation 4.5 vs. $7.2, \mathrm{P}<0.001$ ), short-term postoperative pain and function (VAS at 30th day after operation 3.2 vs. $5.6, \mathrm{P}<0.001$ and ODI $18 \%$ vs. $32 \%, \mathrm{P}<$ 0.001 , respectively), hospital stay $(4.1$ vs. $7.4 \mathrm{~d}$, respectively, $\mathrm{P}=0.015)$, and total blood losses (230 vs. $620 \mathrm{~mL}$, respectively, $\mathrm{P}<0.0001)$. In contrast, there are findings in their study that divert from other evidence in the literature. Park and Ha , [11] as well as [12] report significantly higher surgical time with minimally invasive procedure.

As regard Pain improvement of the studied group show that $18(90 \%)$ had pain improving and $2(10 \%)$ had no improving.

Our results are supported by study of [13] as they mentioned that lumbar pain improved in $83.5 \%$ of patients compared to $90 \%$ lumbar pain improvement in [8] study.

Z. Audat et al.[14] reported that pain symptoms relieved in $70 \%$ of 81 patients, and good outcomes were reported in $80 \%$ of the patients. Show that $17(85 \%)$ had good rate of satisfaction and 3(15\%) had poor satisfaction. Pain improvement of the studied group show that $18(90 \%)$ had pain improving and $2(10 \%)$ had no improving. As regard return to work of the studied group show that $1(5 \%)$ 
didn't return to work, $15(75 \%)$ return to their work and $4(20 \%)$ return to their work but with light work.

Our results are supported by study of [15] as they reported in their study underwent TLIF surgery that fusion rate radiologically was $95 \%$ of cases and good to excellent clinical outcome was achieved in $88 \%$ of cases. In the study of [8], fusion rate was $90 \%$ and improvement in clinical symptoms was $90 \%$ of cases.

C.L. Goldstein et al. [16] found a dural injury rate of $5.4 \%$, graft malposition of $4.4 \%$, screw mal-position of $2.6 \%$, neurologic deficit and nerve injury of $3.8 \%$, reoperation ratio of $3.3 \%$, and reoperation for graft malposition of $1.8 \%$ for PLIF and TLIF procedures.

K. Asil et al. [17] reported that in his study overall complication rate was $23.9 \%$, dural injury rate was $9.9 \%$, graft mal-position rate was $2.82 \%$, and the screw malposition rate was $4.23 \%$.

Regarding Z. Audat et al. [14] there was a significant decrease in the ODI (Oswestry Disability Index) scores over time $(\mathrm{p}<0.005)$ but no significant difference among the groups at different follow-up times. Radiographic fusion rates for Groups I, II and III were $88 \%, 88.9 \%$ and $91.9 \%$, respectively.

F. Shunwu et al. [12] reported that the minimally invasive TLIF group was found to have reduced blood loss, fewer transfusions, less postoperative back pain, lower serum creatine kinase on the third postoperative day, a shorter time to ambulation, and a briefer hospital stay. The Oswestry Disability Index and Visual Analog Scale scores were significantly lower in the minimally invasive group during follow-up. However, the open group had a shorter operative duration. The complications in the 2 groups were similar, but 2 cases of screw malposition occurred in the minimally invasive group.

\section{Conclusion}

TLIF is a technique which offers a simple, safe and effective treatment for degenerative lumbar spine disorders with great improvement of life quality of cases with surgery satisfaction.

\section{References}

[1] H.G. Blume, C.H. Rojas , Unilateral lumbar interbody fusion (posterior approach) utilizing dowel graft. J Neurol Orthop Surg. Vol. 25 (2), PP.171175,1981 .

[2] J. Harms, H. olinger, A one-stager procedure in operative treatment of spondylolistheses: dorsal traction-reposition and anterior fusion (author's transl). Zeitschrift fur Orthopadie und ihre Grenzgebiete, Vol.120(3), PP. 343-347,1982.

[3] S. Lauber, T.L. Schulte, U. Liljenqvist, Clinical and radiologic 2-4-year results of transforaminal lumbar interbody fusion in degenerative and isthmic spondylolisthesis grades 1 and 2 ,Vol. 28(31), PP.1693-1698.

[4] D.K. Resnick, T.F. Choudhri, A.T. Dailey, , Guidelines for the performance of fusion procedures for degenerative disease of the lumbar spine. Part 8: lumbar fusion for disc herniation and radiculopathy. $\mathrm{J}$ Neurosurg Spine, Vol. 30(2), PP.673-8,2005.

[5] P.V. Mummaneni, R.W. Haid, G.E. Rodts , Lumbar interbody fusion: state-of-the-art technical advances. Invited submission from the Joint Section Meeting on Disorders of the Spine and Peripheral Nerves, March. J Neurosurg Spine, Vol.50(1),PP.24-30, 2004.

[6] J.W. Frymoyer, M.H. Pope, J.H. Clements, Risk factors in low back pain. An epidemiological survey. J Bone Joint Surg Am, Vol. 65 (22),PP. 213-8, 1983.

[7] G.B. Andersson, Epidemiological features of chronic low back pain. Lancet,Vol.Vol. 14, PP. 354 581,1999 .

[8] M. Mohammad Hassaan, I. Ali Nassar, A. Abd AlAleem Alsebaie, transforaminal lumbar interbody fusion in management of degenerative lumbar spine disorders. Al-azhar medical journal, Vol.45(1),PP. 161-170, 2016.

[9] S.C. Humphreys, S.D. Hodges, A.G. Patwardhan , Comparison of posterior and transforaminal approaches to lumbar interbody fusion. Spine, Vol.26 PP. 567-571, 2001.

[10] G. B. Brodano , K. Martikos, F. Lolli , Transforaminal lumbar interbody fusion in degenerative disk disease and spondylolisthesis grade I. Journal of Spinal Disorders and Techniques, Vol.28(10), PP.E559-E564,2015.

[11] Y. Park, Y.W. Ha , Comparison of one-level posterior lumbar interbody fusion performed with a minimally invasive approach or a traditional open approach. Spine; 32:537-543,2007.

[12] F. Shunwu, Z. Xing, Z. Fengdong Minimally invasive trans foraminal lumbar interbody fusion for the treatment of degenerative lumbar diseases. Spine , Vol.35, PP.1615-1620,2010.

[13] Deng-Lu Yan, Fu Xing Pei, L. I. Jian ,Comparative study of PILF and TLIF treatment in adult degenerative spondylolisthesis. Eur. Spine , Vol.17, PP. 1311- 1316,2008.

[14] Z. Audat, O. Moutas, K. Yousef ,Comparison of clinical and radiological results of posterolateral fusion, posterior lumbar interbody fusion and tranforaminal lumbar interbody fusion techniques in the treatment of degenerative lumbar spine. Singapore Med , Vol. 53, PP.183-187,2012.

[15] T. Lowe , A. Tahermia , Unilateral transforaminal posterior lumbar interbody fusion procedure. Orthopaedics , Vol. 25, PP. 1179-1183,2002.

[16] C.L. Goldstein, K. Macwan, K. Sundararajan Comparative outcomes of minimally invasive surgery for posterior lumbar fusion: a systematic review. Clin Orthop Relat Res, Vol. 472, PP.1727-1737,2014

[17] K. Asil , C. Yaldiz , Retrospective Comparison of Radiological and Clinical Outcomes of PLIF and TLIF Techniques in Patients Who Underwent Lumbar Spinal Posterior Stabilization. Medicine (Baltimore), Vol. 95(17), PP. e3235,2016. 